\title{
HADAMARD TYPE OPERATORS ON TEMPERATE DISTRIBUTIONS
}

\author{
Dietmar Vogt
}

\begin{abstract}
We study Hadamard operators on $\mathscr{S}^{\prime}\left(\mathbb{R}^{d}\right)$ and give a complete characterization. They have the form $L(S)=S \star T$ where $\star$ means the multiplicative convolution and $T \in \mathscr{O}_{H}^{\prime}\left(\mathbb{R}_{*}^{d}\right)$, the space of distributions which are $\theta$-rapidly decreasing in infinity and at the coordinate hyperplanes. To show this we study and characterize convolution operators on the space $Y\left(\mathbb{R}^{d}\right)$ of exponentially decreasing $C^{\infty}$-functions on $\mathbb{R}^{d}$. We use this and the exponential transformation to characterize the Hadamard operators on $\mathscr{S}^{\prime}(Q), Q$ the positive quadrant, and this result we use as a building block for our main result.
\end{abstract}

In the present note we study Hadamard operators on $\mathscr{S}^{\prime}\left(\mathbb{R}^{d}\right)$, that is, continuous linear operators on $\mathscr{S}^{\prime}\left(\mathbb{R}^{d}\right)$ which admit all monomials as eigenvectors and we give a complete characterization. Operators of Hadamard type have attracted some attention in recent times. Such operators on $C^{\infty}\left(\mathbb{R}^{d}\right)$ have been studied and characterized in [10, 13], on $\mathscr{A}(\mathbb{R})$ in [1, 2, 3] and on $\mathscr{A}\left(\mathbb{R}^{d}\right)$ in [5]. There you find also references to the long history of such problems. Their surjectivity on $C^{\infty}\left(\mathbb{R}^{d}\right)$ has been characterized in 4 . Since it can be shown that Hadamard operators commute with dilations our problem is, by duality, closely related to the study of continuous linear operators in $\mathscr{S}\left(\mathbb{R}^{d}\right)$ which commute with dilations. In a first step we study such operators on $\mathscr{S}(Q), Q=] 0,+\infty\left[{ }^{d}\right.$. By means of the exponential transformation this can be transferred to the study of convolution operators on the space $Y\left(\mathbb{R}^{d}\right)$ of $C^{\infty}$-functions on $\mathbb{R}^{d}$ with exponential decay.

In a first part of the paper we study such operators and give a complete characterization in terms of the class $\mathscr{O}_{Y}^{\prime}\left(\mathbb{R}^{d}\right)$ of exponentially decreasing distributions, which is similar to the class $\mathscr{O}_{C}^{\prime}$ of L. Schwartz of rapidly decreasing distributions,

2010 Mathematics Subject Classification. Primary: 46F10. Secondary: 47B38,44A35,46E10.

Key words and phrases: Hadamard operators, temperate distributions, convolution opera-. tors, $C^{\infty}$-functions of exponential decay. 
which are the convolution-multipliers in $\mathscr{S}\left(\mathbb{R}^{d}\right)$. We study the class $\mathscr{O}_{Y}^{\prime}\left(\mathbb{R}^{d}\right)$ and these results are of independent interest.

By means of the exponential transformation we obtain a description of the operators on $\mathscr{S}^{\prime}(Q)$ which commute with dilations in $Q$. They have the form $\varphi \mapsto T_{x} \varphi(x y)$ where $T$ is a distribution in $\mathscr{O}_{H}^{\prime}(Q)$. These are the exponential transforms of $\mathscr{O}_{Y}^{\prime}\left(\mathbb{R}^{d}\right)$, we call them $\theta$-rapidly decreasing distributions on $Q$. The class $\mathscr{O}_{H}^{\prime}\left(\mathbb{R}^{d}\right)$ first appeared in [1] where the Hadamard operators in $\mathscr{D}^{\prime}\left(\mathbb{R}^{d}\right)$ were described. For a more detailed study of this class and examples see [11, §3].

From there we obtain our main result: The Hadamard operators on $\mathscr{S}^{\prime}\left(\mathbb{R}^{d}\right)$ have the form $S \mapsto S \star T$ where $T \in \mathscr{O}_{H}^{\prime}\left(\mathbb{R}_{*}^{d}\right)$ the class of distributions on $\mathbb{R}^{d}$ which are $\theta$-rapidly decreasing in infinity and at the coordinate hyperplanes. It is a subclass of $\mathscr{O}_{H}^{\prime}\left(\mathbb{R}^{d}\right)$, known from [11].

We use standard notation of Functional Analysis, in particular, of distribution theory. For unexplained notation we refer to [6], [8], [9], [7].

\section{Preliminaries}

We use the following notation $\partial_{j}=\partial / \partial x_{j}, \theta_{j}=x_{j} \partial_{j}$. For a multiindex $\alpha \in \mathbb{N}_{0}^{d}$ we set $\partial^{\alpha}=\partial_{1}^{\alpha_{1}} . . \partial_{d}^{\alpha_{d}}$, likewise for $\theta^{\alpha} .1$ denotes the vector $(1, \ldots, 1)$. For vectors $x, y \in \mathbb{R}^{d}$ we will use the definition $x y=\left(x_{1} y_{1}, \ldots x_{d} y_{d}\right)$. This will hold except for obvious cases like in the formula for the Fourier transform.

For a polynomial $P(z)=\sum_{\alpha} c_{\alpha} z^{\alpha}$ we consider the Euler operator $P(\theta)=\sum_{\alpha} c_{\alpha} \theta^{\alpha}$ and also the operator $P(\partial)$, defined likewise. The dual operator of $P(\theta)$ is $P\left(\theta^{*}\right)$ where $\theta^{*}=-\theta-1$, hence also an Euler operator.

For $a \in \mathbb{R}_{*}^{d}$ the dilation operator $D_{a}$ is defined by $\left(D_{a} T\right) \varphi=\left|a_{1} \cdots a_{d}\right|^{-1} T_{\xi} \varphi(\xi / a)$. For the distribution $x^{\alpha} \in \mathscr{S}^{\prime}\left(\mathbb{R}^{d}\right)$ this yields $D_{a} x^{\alpha}=(a x)^{\alpha}$. For $e \in\{-1,+1\}^{d}$ this definition simplifies to $\left(D_{e} T\right) \varphi=T_{\xi} \varphi(e \xi)$. These operators are called reflections .

For basic properties of Hadamard operators see [11]. They are a closed commutative sub-algebra of $L\left(\mathscr{S}^{\prime}\left(\mathbb{R}^{d}\right)\right)$. Euler operators and dilations are of Hadamard type, Therefore they commute with all Hadamard operators. On the other hand we have:

Lemma 1.1 If $L \in L\left(\mathscr{S}^{\prime}\left(\mathbb{R}^{d}\right)\right)$ commutes with $\theta_{j}$ for all $j$ and with all reflections then it is a Hadamard operator.

Proof: We set $T=L\left(x^{\alpha}\right)$ and have to show that $T \in \operatorname{span}\left\{x^{\alpha}\right\}$. Since $L$ commutes with $\theta_{j}$ we obtain $\theta_{j} T=\alpha_{j} T$. By use of the exponential transformation we obtain for $Q$ and likewise for all quadrants $Q_{e}=e Q$ that $T=c_{e} x^{\alpha}$ on $Q_{e}$, 
with constants $c_{e}$. Since $L$ commutes with reflections all $c_{e}$ must be equal and we have $T=c x^{\alpha}$ on $\mathbb{R}_{*}^{d}$.

We set $S=T-c x^{\alpha}$. Then $\operatorname{supp} S \subset Z_{0}=\left\{\xi: \xi_{1} \cdots \xi_{d}=0\right\}$ and $\theta_{j} S=\alpha_{j} S$. Since $S$ is of finite order there is $\beta \in \mathbb{N}^{d}$ such that $x^{\beta} S=0$. We have

$$
\partial_{j}\left(x^{\beta} S\right)=\beta_{j} x^{\beta^{\prime}} S+x^{\beta^{\prime}} \theta_{j} S=\left(\beta_{j}+\alpha_{j}\right) x^{\beta^{\prime}} S
$$

where $\beta^{\prime}=\left(\beta_{1}, \ldots, \beta_{j}-1, \ldots, \beta_{d}\right)$. Repeating this we obtain:

$$
0=\partial^{\beta}\left(x^{\beta} S\right)=b S
$$

with $b \neq 0$. Therefore $S=0$, that is, $L\left(x^{\alpha}\right)=c x^{\alpha}$.

We set for $x \in \mathbb{R}^{d}$

$$
\operatorname{Exp}(x)=\left(\exp \left(x_{1}\right), . ., \exp \left(x_{d}\right)\right)
$$

Exp is a diffeomorphism from $\mathbb{R}^{d}$ onto $Q:=(0,+\infty)^{d}$. Therefore

$$
C_{\text {Exp }}: f \longrightarrow f \circ \operatorname{Exp}
$$

is a linear topological isomorphism from $C^{\infty}(Q)$ onto $C^{\infty}\left(\mathbb{R}^{d}\right)$. For $f \in C^{\infty}(Q)$ we have $P(\partial)(f \circ \operatorname{Exp})=(P(\theta) f) \circ \operatorname{Exp}$ that is $P(\partial) \circ C_{\operatorname{Exp}}=C_{\operatorname{Exp}} \circ P(\theta)$. In this way the study of Hadamard operators on $Q$ can be reduced to the study of operators on $\mathbb{R}^{d}$. This has be done in 13 for $C^{\infty}(Q)$. We apply the same argument to the space $\mathscr{S}(Q)$ where $\mathscr{S}(Q)=\left\{f \in \mathscr{S}\left(\mathbb{R}^{d}\right): \operatorname{supp} f \subset \bar{Q}\right\}$.

As usual $\mathscr{S}\left(\mathbb{R}^{d}\right)$ denotes the Schwartz space of rapidly decreasing $C^{\infty}$-functions on $\mathbb{R}^{d}$, its dual $\mathscr{S}^{\prime}\left(\mathbb{R}^{d}\right)$ the space of temperate distributions. We consider is subspace $\mathscr{S}(Q)$ and its dual $\mathscr{S}^{\prime}(Q)$.

We recall the following definitions of [9, Chap. VI, §8]: $\mathscr{B}^{\prime}$ denotes the dual of the space of $C^{\infty}$-space which are bounded including all derivatives and $\mathscr{D}_{L_{1}}^{\prime}$ the dual of the space of $C^{\infty}$-space such that all derivatives are in $L_{1}\left(\mathbb{R}^{d}\right)$.

\section{Convolution operators on $C^{\infty}$-functions with exponential decay}

We start with studying convolution operators on the space of $C^{\infty}$-functions with exponential decay on $\mathbb{R}^{d}$ and its dual. We will transfer our results by the exponential diffeomorphism to results on Hadamard operators on $\mathscr{S}^{\prime}(Q)$ and use this as building blocks to study Hadamard operators on $\mathscr{S}^{\prime}\left(\mathbb{R}^{d}\right)$. We set

$$
\begin{aligned}
Y\left(\mathbb{R}^{d}\right) & :=\left\{f \in C^{\infty}\left(\mathbb{R}^{d}\right): \sup _{x}\left|f^{(\alpha)}(x)\right| e^{k|x|}<\infty \text { for all } \alpha \text { and } k \in \mathbb{N}\right\} \\
& =\left\{f \in C^{\infty}\left(\mathbb{R}^{d}\right): \sup _{x}\left|f^{(\alpha)}(x)\right| e^{x \eta}<\infty \text { for all } \alpha \text { and } \eta \in \mathbb{R}^{d}\right\}
\end{aligned}
$$


with its natural topology.

Then $Y\left(\mathbb{R}^{d}\right)$ is a Fréchet space, closed under convolution and $P(\partial)$ is a continuous linear operator in $Y\left(\mathbb{R}^{d}\right)$ for every polynomial $P$. $\mathscr{D}\left(\mathbb{R}^{d}\right) \subset Y\left(\mathbb{R}^{d}\right)$ as a dense subspace, hence $Y\left(\mathbb{R}^{d}\right)^{\prime} \subset \mathscr{D}^{\prime}\left(\mathbb{R}^{d}\right)$. We obtain (see [14, Lemma 2.1]):

Lemma 2.1 $C_{\operatorname{Exp}}(\mathscr{S}(Q))=Y\left(\mathbb{R}^{d}\right)$.

We set $\omega(x)=\sum_{\eta \in\{-1,+1\}^{d}} e^{\eta x}$. We have $\omega \in C^{\infty}\left(\mathbb{R}^{d}\right)$ and $e^{|x|} \leq \omega(x) \leq 2^{d} e^{|x|}$.

In analogy to [9, Chap. VII, §5, p. 100] we define

Definition $1 T \in \mathscr{O}_{Y}^{\prime}\left(\mathbb{R}^{d}\right)$ if $\omega(k x) T \in \mathscr{B}^{\prime}$ for every $k$.

It is obvious that we might equivalently write $\omega(k x) T \in \mathscr{D}_{L_{1}}^{\prime}$ for every k.

For the following theorem compare [9, Chap. VII, §5, Théorème IX].

Theorem 2.2 For $T \in \mathscr{D}^{\prime}\left(\mathbb{R}^{d}\right)$ the following are equivalent:

1. $T \in \mathscr{O}_{Y}^{\prime}\left(\mathbb{R}^{d}\right)$.

2. For any $k$ there are finitely many functions $t_{\beta}$ such that $e^{k|x|} t_{\beta} \in L_{\infty}\left(\mathbb{R}^{d}\right)$ and such that $T=\sum_{\beta} \partial^{\beta} t_{\beta}$.

3. $T \in Y\left(\mathbb{R}^{d}\right)^{\prime}$ and $T_{x} \varphi(x+y) \in Y\left(\mathbb{R}^{d}\right)$ for all $\varphi \in Y\left(\mathbb{R}^{d}\right)$.

4. $f(y)=T_{x} \varphi(x+y)$ is a exponentially decreasing continuous function (that is $\sup |f(y)| e^{k|y|}<\infty$ for all $\left.k\right)$ for all $\varphi \in \mathscr{D}\left(\mathbb{R}^{d}\right)$.

5. $(\omega(k x) T) * \varphi$ is a continuous bounded function for every $k$ and $\varphi \in \mathscr{D}\left(\mathbb{R}^{d}\right)$.

Proof: $\quad(1) \Rightarrow(2)$ If $\omega(k x) T \in \mathscr{D}_{L_{1}}^{\prime}$ then, by a standard conclusion, there are finitely many functions $\tau_{\beta} \in L_{\infty}\left(\mathbb{R}^{d}\right)$ such that $\omega(k x) T=\sum_{\beta} \partial^{\beta} \tau_{\beta}$. This yields

$$
\begin{aligned}
T \varphi & =(\omega(k x) T)\left(\frac{1}{\omega(k x)} \varphi\right)=\sum_{\beta} \partial^{\beta} \tau_{\beta}\left(\frac{1}{\omega(k x)} \varphi\right) \\
& =\sum_{\beta}(-1)^{|\beta|} \int \tau_{\beta}(x) \partial^{\beta}\left(\frac{1}{\omega(k x)} \varphi(x)\right) d x \\
& =\sum_{\beta}(-1)^{|\beta|} \int \tau_{\beta}(x) \sum_{\alpha \leq \beta} c_{\alpha, \beta}\left(\partial^{\beta-\alpha} \frac{1}{\omega(k x)}\right) \varphi^{(\alpha)}(x) d x \\
& =\sum_{\alpha}\left(\sum_{\beta \geq \alpha}(-1)^{|\beta|} \tau_{\beta}(x) c_{\alpha, \beta} \partial^{\beta-\alpha} \frac{1}{\omega(k x)}\right) \varphi^{(\alpha)}(x) d x \\
& =\left\langle\sum_{\alpha} \partial^{\alpha} t_{\alpha}, \varphi\right\rangle
\end{aligned}
$$


where $\omega(k x) t_{\alpha} \in L_{\infty}\left(\mathbb{R}^{d}\right)$ for all the, finitely many, $\alpha$.

$(2) \Rightarrow(1)$ is straightforward, because we may assume that $T=\partial^{\beta} t_{\beta}$.

$(2) \Rightarrow(3)$ The first part is clear from (2). Assume $T=\partial^{\beta} t_{\beta}, e^{(k+1)|x|}\left|t_{\beta}(x)\right| \in$ $L_{\infty}\left(\mathbb{R}^{d}\right)$. Then

$$
T_{x} \varphi(x+y)=(-1)^{|\beta|} \int t_{\beta}(x) \varphi^{(\beta)}(x+y) d x \in C^{\infty}\left(\mathbb{R}^{d}\right)
$$

and we have

$$
e^{k|y|}\left|\partial_{y}^{\alpha} T_{x} \varphi(x+y)\right| \leq \int e^{k|x|}\left|t_{\beta}(x)\right| e^{k|x+y|}\left|\varphi^{(\alpha+\beta)}(x+y)\right| d x \leq \infty .
$$

If $T \in \mathscr{O}_{Y}^{\prime}\left(\mathbb{R}^{d}\right)$ this holds for all summands in the representation of $T$ with given $k$ and since we have for all $k$ such a representation the claim is proved.

$(3) \Rightarrow(4)$ is obvious.

$(4) \Rightarrow(5)$ For $\varphi \in \mathscr{D}\left(\mathbb{R}^{d}\right)$ and $\eta \in \mathbb{R}^{d}$ we obtain

$\left(\left(e^{\eta x} T\right) * \varphi\right)(y)=T_{x}\left(e^{\eta x} \varphi(y-x)\right)=e^{\eta y} T e^{-\eta(y-x)} \varphi(y-x)=e^{\eta y}\left(T *\left(e^{-\eta x} \varphi\right)\right)(y)$.

Since $e^{-\eta x} \varphi \in \mathscr{D}\left(\mathbb{R}^{d}\right)$ the right hand side is bounded, by (4). Adding over all $\eta \in\{+k,-k\}^{d}$ we obtain the result.

$(5) \Rightarrow(2)$ This follows from Lemma 2.3 .

The following Lemma is essentially an adaptation of [9, Chap. VI, §8, Théorème $\mathrm{XXV}]$.

Lemma 2.3 Let $\omega$ be measurable, $\omega(x)>0$ for all $x \in \mathbb{R}^{d}$. Let $S \in \mathscr{D}^{\prime}\left(\mathbb{R}^{d}\right)$ be a distribution such that $\sup _{x} \omega(x)\left|S_{y} \varphi(x-y)\right|<\infty$ for all $\varphi \in \mathscr{D}\left(\mathbb{R}^{d}\right)$ then there are finitely many measurable functions $\tau_{\beta}$ with $\sup _{x} \omega(x) \tau_{\beta}(x)<\infty$ such that $S=\sum_{\beta} \tau_{\beta}^{(\beta)}$.

Proof: We consider the map $\Psi: \mathscr{D}\left(\mathbb{R}^{d}\right) \rightarrow L_{\infty}\left(\mathbb{R}^{d}\right)$ given by

$$
\Psi(\varphi)=\omega(x) S_{y} \varphi(x-y) .
$$

Because of the Closed Graph Theorem $\Psi$ is continuous. Let $B$ denote the unit ball in $\mathbb{R}^{d}$. Then there is $m \in \mathbb{N}$ such that $\Psi$ restricted to $\mathscr{D}(B)$ extends to a continuous map $\mathscr{D}^{m}(B) \rightarrow L_{\infty}\left(\mathbb{R}^{d}\right)$, where $\mathscr{D}^{m}(B)$ denotes the Banach space of $m$-times continuously differentiable functions with support in $B$. We choose $\gamma \in \mathscr{D}(B), \gamma(x)=1$ in a neighborhood of 0 and set $g=\gamma E \in \mathscr{D}^{m}(B)$ where $E$ is an elementary solution of $\Delta^{k}, k$ large enough. Then $\Psi(g) \in L_{\infty}\left(\mathbb{R}^{d}\right)$ that means $\tau:=S * g$ is a measurable function with $\omega(x)|\tau(x)| \leq C$ for suitable $C$ and we obtain $\Delta^{k} \tau=S+S * \psi$ where $\psi \in \mathscr{D}(B)$. We have $\omega(x)(S * \psi)=\Psi(\psi) \in L_{\infty}\left(\mathbb{R}^{d}\right)$. Therefore the equality $S=\Delta^{k} \tau-S * \psi$ shows the result. 
We have to fix our notation on the convolution of distributions. For distributions $T, S$ and a function $\psi$ we define $(S * T) \psi:=S_{y}\left(T_{x} \psi(x+y)\right)$ whenever this makes sense.

Lemma 2.4 If $T \in \mathscr{O}_{Y}^{\prime}\left(\mathbb{R}^{d}\right)$ and $\varphi \in Y\left(\mathbb{R}^{d}\right)$ then both $T * \varphi \in Y\left(\mathbb{R}^{d}\right)^{\prime}$ and $\varphi * T \in$ $Y\left(\mathbb{R}^{d}\right)^{\prime}$ are defined and equal and we have $T * \varphi=\varphi * T=T_{y} \varphi(x-y) \in Y\left(\mathbb{R}^{d}\right)$. $\varphi \mapsto T * \varphi$ is a continuous linear operator in $Y\left(\mathbb{R}^{d}\right)$.

Proof: The first claim follows from Theorem 2.2. (3), the fact that $Y\left(\mathbb{R}^{d}\right)$ is closed under convolution and, finally, from the representation in Theorem 2.2, (2). The second is then easily shown or follows from the Closed Graph Theorem.

This shows part of the following theorem.

Theorem 2.5 For an operator $L \in L\left(Y\left(\mathbb{R}^{d}\right)\right)$ the following are equivalent:

1. L commutes with translations.

2. There is $T \in \mathscr{O}_{Y}^{\prime}\left(\mathbb{R}^{d}\right)$ such that $L \varphi=T * \varphi$ for all $\varphi \in Y\left(\mathbb{R}^{d}\right)$.

Proof: $(2) \Rightarrow(1)$ is clear, we have to show the converse. We define $T \in Y\left(\mathbb{R}^{d}\right)^{\prime}$ by $T \varphi:=(L \varphi)(0)$. Then by standard arguments we have $(L \varphi)(x)=L(\varphi(\cdot+$ $x))(0)=T_{y} \varphi(y+x)=\check{T} \varphi(x-y)=(\check{T} * \varphi)(x)$. Due to Theorem 2.2, (3) we have $T \in \mathscr{O}_{Y}^{\prime}\left(\mathbb{R}^{d}\right)$, hence also $\check{T} \in \mathscr{O}_{Y}^{\prime}\left(\mathbb{R}^{d}\right)$.

The dual situation is a bit more complicated, since existence of $T * S$ and commutivity is not a priori clear. We define:

$$
\mathscr{O}_{Y}\left(\mathbb{R}^{d}\right):=\left\{f \in C^{\infty}\left(\mathbb{R}^{d}\right): \exists k \in \mathbb{N}_{0} \forall \alpha \in \mathbb{N}_{0}^{d}: \sup _{x \in \mathbb{R}^{d}}\left|f^{(\alpha)}(x)\right| e^{-k|x|}<\infty\right\} .
$$

Equipped with its natural locally convex topology $\mathscr{O}_{Y}\left(\mathbb{R}^{d}\right)$ is the inductive limit of a sequence of Fréchet spaces, that is, an (LF)-space and we have

Lemma 2.6 $\mathscr{O}_{Y}^{\prime}\left(\mathbb{R}^{d}\right)$ is the dual space of $\mathscr{O}_{Y}\left(\mathbb{R}^{d}\right)$. For $S \in Y\left(\mathbb{R}^{d}\right)^{\prime}$ the map $\varphi \mapsto S_{y} \varphi(x+y)$ is a continuous linear map from $Y\left(\mathbb{R}^{d}\right)$ to $\mathscr{O}_{Y}\left(\mathbb{R}^{d}\right)$.

Proof: The first part by use of a standard argument using Theorem 2.2, (2). For the second part we estimate

$$
\begin{aligned}
\left|\partial^{\alpha} S_{y} \varphi(x+y)\right| & =\left|S_{y} \varphi^{(\alpha)}(x+y)\right| \leq C \sup _{y,|\beta| \leq k} \mid \varphi^{(\alpha+\beta)}(x+y) e^{k|y|} \\
& \leq C e^{k|y|} \sup _{\xi,|\gamma| \leq m}\left|\varphi^{(\gamma)}(\xi)\right| e^{k|\xi|}
\end{aligned}
$$

with $C$ and $k$ depending on $S$ and $m=k+|\alpha|$.

We obtain an analogue to Lemma 2.4. 
Lemma 2.7 If $T \in \mathscr{O}_{Y}^{\prime}\left(\mathbb{R}^{d}\right)$ and $S \in Y\left(\mathbb{R}^{d}\right)^{\prime}$ then both $T * S \in Y\left(\mathbb{R}^{d}\right)^{\prime}$ and $S * T \in Y\left(\mathbb{R}^{d}\right)^{\prime}$ are defined and equal. $S \mapsto T * S$ is a continuous linear operator in $Y\left(\mathbb{R}^{d}\right)^{\prime}$.

Proof: The existence of $S * T$ follows from Theorem 2.2, (3), the existence of $T * S$ from Lemma 2.7. $(T * S) \varphi=(S * T) \varphi$ for $\varphi \in \mathscr{D}\left(\mathbb{R}^{d}\right)$ equality follows by direct calculation by use of Theorem 2.2, (2). The continuity of $S \mapsto S * T$ is obvious.

Theorem 2.8 For an operator $L \in L\left(Y\left(\mathbb{R}^{d}\right)^{\prime}\right)$ the following are equivalent:

\section{L commutes with translations.}

2. There is $T \in \mathscr{O}_{Y}^{\prime}\left(\mathbb{R}^{d}\right)$ such that $L(S)=T * S$ for all $S \in Y\left(\mathbb{R}^{d}\right)^{\prime}$.

Proof: $\quad(2) \Rightarrow(1)$ is clear, we have to show the converse. The transpose $L^{*} \in L\left(Y\left(\mathbb{R}^{d}\right)\right)$ also commutes with translation. Note that $Y\left(\mathbb{R}^{d}\right)$ is Montel, hence reflexive. Because of Theorem 2.5, Proof, there is $T \in \mathscr{O}_{Y}^{\prime}\left(\mathbb{R}^{d}\right)$ such that $\left(L^{*}(\varphi)\right)(x)=T_{y} \varphi(x+y)$. So for $S \in Y\left(\mathbb{R}^{d}\right)^{\prime}$ we obtain $\langle L(S), \varphi\rangle=\left\langle S, L^{*}(\varphi)\right\rangle=$ $S_{x}\left(T_{y} \varphi(x+y)\right)=((S * T) \varphi)(x)$.

\section{$3 \quad$ Hadamard operators on $\mathscr{S}^{\prime}(Q)$}

Let $L$ be a Hadamard operator on $\mathscr{S}^{\prime}(Q)$, that is an operator which admits all monomials as eigen-functions. We need some preparations, cf. Section 1 in [11]. For $a \in Q$ we define the dilation $D_{a} \in L\left(\mathscr{S}^{\prime}(Q)\right)$ by

$$
\left(D_{a} T\right) \varphi:=T_{x}\left(\frac{1}{a_{1} \ldots a_{d}} \varphi\left(\frac{x}{a}\right)\right)
$$

for $T \in \mathscr{S}^{\prime}(Q)$ and $\varphi \in \mathscr{S}(Q)$. By direct verification we see that $D_{a} \xi^{\alpha}=a^{\alpha} \xi^{\alpha}$.

Like in [11, Lemma 1.1] we obtain that $L$ commutes with dilations, that is, $D_{a} \circ L=L \circ D_{a}$ for all $a \in Q$.

We set $M=L^{*} \in L(\mathscr{S}(Q))$ and obtain like in [11, Lemma 1.3] that $M$ commutes with with dilations, that is,

$$
M_{\xi}(\varphi(\eta \xi))[x]=(M \varphi)(\eta x)
$$

for all $\varphi \in \mathscr{S}(Q)$ and $\eta \in Q$.

For $\varphi \in \mathscr{S}(Q)$ we define now

$$
T \varphi=(M \varphi)(\mathbf{1})=\left(L \delta_{\mathbf{1}}\right)(\varphi)
$$


Then $T \in \mathscr{S}^{\prime}(Q)$ and for all $\eta \in Q$ we have

$$
(M \varphi)(\eta)=T_{\xi} \varphi(\eta \xi)
$$

We have to determine the set of distributions in $T \in \mathscr{S}^{\prime}(Q)$ such that

$$
T_{\xi} \varphi(\cdot \xi) \in \mathscr{S}(Q) \text { for all } \varphi \in \mathscr{S}(Q)
$$

For $\widetilde{T}=C_{\mathrm{Log}}^{*}(T)$ the condition (2) is equivalent to

$$
\widetilde{T}_{\xi} \psi(\cdot+\xi) \in Y\left(\mathbb{R}^{d}\right) \text { for all } \psi \in Y\left(\mathbb{R}^{d}\right)
$$

which, by Theorem 2.2, is equivalent to $\widetilde{T} \in \mathscr{O}_{Y}^{\prime}\left(\mathbb{R}^{d}\right)$.

In analogy to [11, Definition 3] we define the space $\mathscr{O}_{H}^{\prime}(Q)$ of $\theta$-rapidly decreasing distributions on $Q$.

Definition $2 T \in \mathscr{O}_{H}^{\prime}(Q)$ if for any $k$ there are finitely many functions $t_{\beta}$ such that $\left(|x|^{2 k}+|x|^{-2 k}\right) t_{\beta} \in L_{\infty}(Q)$ and such that $T=\sum_{\beta} \theta^{\beta} t_{\beta}$.

By use of the description in Theorem 2.2, (2), we obtain:

Lemma 3.1 $C_{\operatorname{Exp}}^{*}\left(\mathscr{O}_{Y}^{\prime}\left(\mathbb{R}^{d}\right)\right)=\mathscr{O}_{H}^{\prime}(Q)$.

Hence we obtain the following translation of Theorem 2.2.

Theorem 3.2 For $T \in \mathscr{D}^{\prime}\left(\mathbb{R}^{d}\right)$ the following are equivalent:

1. $T \in \mathscr{O}_{H}^{\prime}(Q)$

2. For any $k$ there are finitely many functions $t_{\beta}$ such that $\left(|x|^{2 k}+|x|^{-2 k}\right) t_{\beta} \in$ $L_{\infty}(Q)$ and such that $T=\sum_{\beta} \theta^{\beta} t_{\beta}$

3. $T \in \mathscr{S}^{\prime}(Q)$ and $T_{x} \varphi(x y) \in \mathscr{S}(Q)$ for all $\varphi \in \mathscr{S}(Q)$.

4. $f(y)=T_{x} \varphi(x y)$ is a rapidly decreasing continuous function (that is $\sup |f(y)|$ $\left(|y|^{2 k}+|y|^{-2 k}\right)<\infty$ for all $k$ ) for all $\varphi \in \mathscr{D}(Q)$.

5. $\left(\left(|x|^{2 k}+|x|^{-2 k}\right) T\right) \star \varphi$ is a continuous bounded function for every $k$ and $\varphi \in \mathscr{D}(Q)$.

We have obtained the following.

Theorem 3.3 L Hadamard operator on $\mathscr{S}^{\prime}(Q)$ if and only if there is $T \in \mathscr{O}_{H}^{\prime}(Q)$ such that $L(S)=S \star T$ for all $T \in \mathscr{S}^{\prime}(Q)$.

Here $\langle S \star T, \varphi\rangle=S_{x}\left(T_{y} \varphi(x y)\right.$ for all $\varphi \in \mathscr{S}(Q)$. 


\section{$4 \quad$ Hadamard operators on $\mathscr{S}^{\prime}\left(\mathbb{R}^{d}\right)$}

Let now $L$ be a Hadamard operator on $\mathscr{S}^{\prime}\left(\mathbb{R}^{d}\right)$ and $M=L^{*} \in L\left(\mathscr{S}\left(\mathbb{R}^{d}\right)\right)$ and obtain like in [11, Lemma 1.3] that $M$ commutes with with dilations, that is,

$$
M_{\xi}(\varphi(\eta \xi))[x]=(M \varphi)(\eta x)
$$

for all $\varphi \in \mathscr{S}\left(\mathbb{R}^{d}\right)$ and $\eta \in \mathbb{R}_{*}^{d}$.

For $\varphi \in \mathscr{S}\left(\mathbb{R}^{d}\right)$ we define now

$$
T \varphi=(M \varphi)(\mathbf{1})=\left(L \delta_{\mathbf{1}}\right)(\varphi) .
$$

Then $T \in \mathscr{S}^{\prime}\left(\mathbb{R}^{d}\right)$ and for all $\eta \in \mathbb{R}_{*}^{d}$ we have

$$
(M \varphi)(\eta)=T_{\xi} \varphi(\eta \xi)
$$

We have to determine the set of distributions in $T \in \mathscr{S}^{\prime}\left(\mathbb{R}^{d}\right)$ such that $T_{\xi} \varphi(\cdot \xi)$, $\xi \in \mathbb{R}_{*}^{d}$, extends to a function in $\mathscr{S}\left(\mathbb{R}^{d}\right)$ for all $\varphi \in \mathscr{S}\left(\mathbb{R}^{d}\right)$.

We want to use the results of Section 3. We denote by $H_{j}, j=1, . ., d$, the coordinate hyperplanes and set $Z_{0}=\bigcup_{j} H_{j}$.

$$
\mathscr{S}\left(\mathbb{R}_{*}^{d}\right)=\left\{\varphi \in \mathscr{S}\left(\mathbb{R}^{d}\right): \varphi \text { flat on } Z_{0}\right\} \text {. }
$$

We will show that $M\left(\mathscr{S}\left(\mathbb{R}_{*}^{d}\right)\right) \subset \mathscr{S}\left(\mathbb{R}_{*}^{d}\right)$. For that it suffices to show that $L\left(\mathscr{S}^{\prime}\left(Z_{0}\right)\right) \subset \mathscr{S}^{\prime}\left(Z_{0}\right)$. Here $\mathscr{S}^{\prime}\left(Z_{0}\right)$ denotes the temperate distributions with support in $Z_{0}$.

By $\mathcal{F}$ we denote the Fourier transform and remark that for all $j$

$$
\theta_{j} \circ \mathcal{F}=\mathcal{F} \circ \theta_{j}^{*}, \quad \theta_{j}^{*} \circ \mathcal{F}=\mathcal{F} \circ \theta_{j} .
$$

We set $\widetilde{L}=\mathcal{F} \circ L \circ \mathcal{F}^{-1}$ and since $\theta_{j}^{*}$ commutes with $L$ we conclude by use of (7) that $\widetilde{L}$ commutes with $\theta_{j}$ for all $j$. By straightforward calculation we see that $\widetilde{L}$ commutes with all reflections. By Lemma 1.1 this implies that $\widetilde{L}$ is a Hadamard operator. We have $L=\mathcal{F}^{-1} \circ \widetilde{L} \circ \mathcal{F}$.

We have $\mathcal{F}\left(\delta^{(\alpha)}\right)=i^{\alpha}(2 \pi)^{-d / 2} x^{\alpha}$, hence $\widetilde{L}\left(\mathcal{F} \delta^{(\alpha)}\right)=\widetilde{m}_{\alpha} \mathcal{F} \delta^{(\alpha)}$. Finally we obtain

$$
L\left(\delta^{(\alpha)}\right)=\widetilde{m}_{\alpha} \delta^{(\alpha)},
$$

where $\widetilde{L}\left(x^{\alpha}\right)=\widetilde{m}_{\alpha} x^{\alpha}$.

ExAmple: $L=\theta$ then $\widetilde{L}=\theta^{*}=-\theta-1$. Since $\widetilde{m}_{k}=-k-1$ we obtain $\theta \delta^{(k)}=(-k-1) \delta^{(k)}$ which, of course, can be verified by direct calculation.

In fact, we will need this result only for $d=1$. We set $x=\left(x_{1}, x^{\prime}\right), x^{\prime}=\left(x_{2}, . ., x_{d}\right)$ and consider distributions of the form $T_{\alpha}=\delta^{(\alpha)}\left(x_{1}\right) \otimes S\left(x^{\prime}\right), S \in \mathscr{S}^{\prime}\left(\mathbb{R}^{d-1}\right)$. 
We fix $\alpha^{\prime}=\left(\alpha_{2}, . ., \alpha_{d}\right)$ and $\psi \in \mathscr{D}\left(\mathbb{R}^{d-1}\right)$. For $T \in \mathscr{S}^{\prime}\left(\mathbb{R}^{d}\right)$ and $\varphi \in \mathscr{S}(\mathbb{R})$ we set $\left(R_{\psi} T\right) \varphi:=T\left(\varphi\left(x_{1}\right) \psi\left(x^{\prime}\right)\right.$. This defines a map $R_{\psi}: \mathscr{S}^{\prime}\left(\mathbb{R}^{d}\right) \rightarrow \mathscr{S}^{\prime}(\mathbb{R})$.

For $U \in \mathscr{S}^{\prime}(\mathbb{R})$ we set $L_{1}(U):=\left(R_{\psi} \circ L\right)\left(U \otimes x^{\alpha^{\prime}}\right) \in \mathscr{S}^{\prime}(\mathbb{R})$. We obtain for $\alpha \in \mathbb{N}_{0}$ and $\hat{\alpha}=\left(\alpha, \alpha^{\prime}\right)$

$$
L_{1}\left(x^{\alpha}\right)=R_{\psi}\left(L x^{\hat{\alpha}}\right)=R_{\psi}\left(m_{\hat{\alpha}} x^{\hat{\alpha}}\right)=m_{\hat{\alpha}} \int \xi^{\alpha^{\prime}} \psi(\xi) d \xi x^{\alpha} .
$$

Hence $L_{1}$ is a Hadamard operator on $\mathscr{S}^{\prime}(\mathbb{R})$ and, by $(8), \delta^{(\alpha)}$ is an eigenvector of $L_{1}$. This means $L_{1}\left(\delta^{(\alpha)}\right)=\mu_{\alpha} \delta^{(\alpha)}$, hence $(-1)^{\alpha} \mu_{\alpha} \varphi^{(\alpha)}(0)=T\left(\varphi\left(x_{1}\right), \psi\left(x^{\prime}\right)\right)$ for all $\varphi \in \mathscr{S}(\mathbb{R})$ where $T=L\left(\delta^{(\alpha)} \otimes x^{\alpha^{\prime}}\right)$.

We choose $\chi \in \mathscr{D}(\mathbb{R}), \chi=0$ in a neighborhood of 0 , and set $\varphi_{\alpha}(x)=\frac{x^{\alpha}}{\alpha !} \chi(x)$. Then $\mu_{\alpha}=(-1)^{\alpha} T\left(\varphi_{\alpha}\left(x_{1}\right), \psi\left(x^{\prime}\right)\right)$. Setting $\mu_{\alpha}(\psi)=T\left(\varphi_{\alpha}\left(x_{1}\right), \psi\left(x^{\prime}\right)\right)$ we obtain a distribution $\mu_{\alpha} \in \mathscr{S}^{\prime}\left(\mathbb{R}^{d-1}\right)$ such that

$$
L\left(\delta^{(\alpha)} \otimes x^{\alpha^{\prime}}\right)=\delta^{(\alpha)} \otimes \mu_{\alpha}
$$

We fix $\alpha \in \mathbb{N}_{0}$ and we have shown, that $x^{\alpha^{\prime}} \in\left\{S \in \mathscr{S}^{\prime}\left(\mathbb{R}^{d-1}\right): L\left(T_{\alpha}\right) \in\right.$ $\left.\delta^{(\alpha)} \otimes \mathscr{S}\left(\mathbb{R}^{d-1}\right)\right\}$ for all $\alpha^{\prime} \in \mathbb{N}_{0}^{d-1}$. Since this set is a closed linear subspace of $\mathscr{S}^{\prime}\left(\mathbb{R}^{d-1}\right)$ we have shown: $L\left(T_{\alpha}\right) \in \delta^{(\alpha)} \otimes \mathscr{S}\left(\mathbb{R}^{d-1}\right)$ for all $S \in \mathscr{S}\left(\mathbb{R}^{d-1}\right)$.

Distributions $T \in \mathscr{S}^{\prime}\left(H_{1}\right)$ have the form

$$
T=\sum_{\alpha=0}^{m} \delta^{(\alpha)}\left(x_{1}\right) \otimes S_{\alpha}\left(x^{\prime}\right)
$$

(cf. [8, Chap III, Théorème XXXVI]). So we have shown $L\left(\mathscr{S}^{\prime}\left(H_{1}\right)\right) \subset \mathscr{S}^{\prime}\left(H_{1}\right)$. By an analogous argument this holds also for $H_{j}, j=2, . ., d$.

Since $\mathscr{S}^{\prime}\left(Z_{0}\right)=\sum_{j=1}^{d} \mathscr{S}^{\prime}\left(H_{j}\right)$ (see [14, Lemma 3.3]) we have shown:

Lemma $4.1 L\left(\mathscr{S}^{\prime}\left(Z_{0}\right)\right) \subset \mathscr{S}^{\prime}\left(Z_{0}\right)$.

As an immediate consequence we obtain:

Proposition $4.2 M\left(\mathscr{S}\left(\mathbb{R}_{*}^{d}\right)\right) \subset \mathscr{S}\left(\mathbb{R}_{*}^{d}\right)$.

We put $M_{+}(\varphi)=\left.M(\varphi)\right|_{Q}$ for $\varphi \in \mathscr{S}(Q)$. Then $M_{+} \in L(\mathscr{S}(Q))$ and $L_{+}:=M_{+}^{*} \in$ $L\left(\mathscr{S}^{\prime}(Q)\right)$ is a Hadamard operator. From Theorem 3.2 we get $T_{+}=L_{+}(\mathbf{1})=$ $\left.T\right|_{\mathscr{S}(Q)} \in \mathscr{O}_{H}^{\prime}(Q)$.

Clearly we can do this for all quadrants $Q_{e}=\{x:$ ex $\in Q\}$. We set $M_{e}(\varphi)=$ $\left.M(\varphi)\right|_{Q}$ for $\varphi \in \mathscr{S}\left(Q_{e}\right)$ and $T_{e}(\varphi)=\left(M_{e} \varphi\right)(\mathbf{1})$ for $\varphi \in \mathscr{S}\left(Q_{e}\right)$. By the same arguments as before we obtain that $T_{e} \in \mathscr{O}_{H}^{\prime}\left(Q_{e}\right)$ (defined in obvious analogy).

In analogy to Definition 2 we define the space of distributions on $\mathbb{R}^{d}$, which are $\theta$-rapidly decreasing in infinity and at the coordinate hyperplanes. 
Definition $3 T \in \mathscr{O}_{H}^{\prime}\left(\mathbb{R}_{*}^{d}\right)$ if for any $k$ there are finitely many functions $t_{\beta}$ such that $\left(|x|^{2 k}+|x|^{-2 k}\right) t_{\beta} \in L_{\infty}\left(\mathbb{R}^{d}\right)$ and such that $T=\sum_{\beta} \theta^{\beta} t_{\beta}$.

Then we have for $T$ as defined in (5) :

Lemma 4.3 $\widetilde{T}:=\sum_{e} T_{e} \in \mathscr{O}_{H}^{\prime}\left(\mathbb{R}_{*}^{d}\right)$ and $\left.T\right|_{\mathscr{S}\left(\mathbb{R}_{*}^{d}\right)}=\widetilde{T}$.

For $T \in \mathscr{O}_{H}^{\prime}\left(\mathbb{R}_{*}^{d}\right)$ and $\varphi \in \mathscr{S}\left(\mathbb{R}^{d}\right)$ we define $\left(M_{T} \varphi\right)(x)=T_{\xi} \varphi(\xi x)$ which is defined for all $x \in \mathbb{R}^{d}$.

Lemma 4.4 $M_{T}$ is a continuous linear operator in $\mathscr{S}\left(\mathbb{R}^{d}\right), L_{T}:=M_{T}^{*}$ is a Hadamard operator.

Proof: We have to estimate $\Psi(x):=x^{\gamma}\left(M_{T} \varphi\right)^{(\alpha)}(x)$. We first recall that $\theta_{\xi}^{*} \varphi(\xi x)=\left(\theta^{*} \varphi\right)(\xi x)$ and $\left(\theta^{*}\right)^{\beta} \xi^{\alpha} \varphi(\xi)=\xi^{\alpha} \sum_{\nu \leq \beta} p_{\nu}(\xi) \varphi^{(\nu)}(\xi)=: \xi^{\alpha} \psi(\xi)$, where the $p_{\nu}$ are polynomials.

We choose $k=k(\alpha-\gamma)$ large enough and obtain

$$
\begin{aligned}
\Psi(x) & =x^{\gamma} \int \theta^{\beta} \tau_{\beta}(\xi) \xi^{\alpha} \varphi^{(\alpha)}(\xi x) d \xi=x^{\gamma-\alpha} \int \theta^{\beta} \tau_{\beta}(\xi)(\xi x)^{\alpha} \varphi^{(\alpha)}(\xi x) d \xi \\
& =x^{\gamma-\alpha} \int \tau_{\beta}(\xi)(\xi x)^{\alpha} \psi(\xi x) d \xi=\int \tau_{\beta}(\xi) \xi^{\alpha-\gamma}(\xi x)^{\gamma} \psi(\xi x) d \xi
\end{aligned}
$$

and therefore

$$
\|\Psi\|_{\gamma, \alpha} \leq\left(\int\left|\tau_{\beta}(\xi) \xi^{\alpha-\gamma}\right| d \xi\right)\|\varphi\| .
$$

Here $\|\varphi\|:=\sup _{x}\left|x^{\gamma} \psi(x)\right|$ is a continuous semi-norm on $\mathscr{S}\left(\mathbb{R}^{d}\right)$. This shows the first part of the claim.

For the second part we have to study $\int x^{\gamma}\left(M_{T} \varphi\right)(x) d x$. We obtain

$$
\begin{aligned}
\int x^{\gamma}\left(\int \theta^{\beta} \tau_{\beta}(\xi) \varphi(\xi x) d \xi\right) d x & =\int \theta^{\beta} \tau_{\beta}(\xi) \xi^{-\gamma}\left(\int(\xi x)^{\gamma} \varphi(\xi x) d x\right) d \xi \\
& =\left(\int \theta^{\beta} \tau_{\beta}(\xi) \xi^{-\gamma-1} d \xi\right) \int x^{\gamma} \varphi(x) d x \\
& =(-\gamma-\mathbf{1})^{\beta}\left(\int \tau_{\beta}(\xi) \xi^{-\gamma-1} d \xi\right) \int x^{\gamma} \varphi(x) d x \\
& =\int\left(m_{\gamma} x^{\gamma}\right) \varphi(x) d x
\end{aligned}
$$

We have shown that $L_{T} x^{\gamma}=m_{\gamma} x^{\gamma}$ and this completes the proof.

For $S \in \mathscr{S}^{\prime}\left(\mathbb{R}^{d}\right), T \in \mathscr{O}_{H}^{\prime}\left(\mathbb{R}_{*}^{d}\right)$ we define $S \star T \in L\left(\mathscr{S}^{\prime}\left(\mathbb{R}^{d}\right)\right)$ by

$$
(S \star T)(\varphi)=S_{x}\left(T_{\xi} \varphi(\xi x)\right) \text { for all } \varphi \in \mathscr{S}\left(\mathbb{R}^{d}\right) .
$$

The following is the main result of this paper. 
Theorem 4.5 1. For every $T \in \mathscr{O}_{H}^{\prime}\left(\mathbb{R}_{*}^{d}\right)$ the map $S \mapsto S \star T$ is a Hadamard operator on $\mathscr{S}^{\prime}\left(\mathbb{R}^{d}\right)$.

2. For every Hadamard operator $L$ on $\mathscr{S}^{\prime}\left(\mathbb{R}^{d}\right)$ there is $T \in \mathscr{O}_{H}^{\prime}\left(\mathbb{R}_{*}^{d}\right)$ such that $L(S)=S \star T$ for all $S \in \mathscr{S}^{\prime}\left(\mathbb{R}^{d}\right)$.

Proof: The first part is Lemma 4.4. For the second part we choose $T=L\left(\delta_{1}\right)$, then $(M \varphi)(\eta)=T_{\xi} \varphi(\eta \xi)$ for all $\eta \in \mathbb{R}_{*}^{d}$ (see (6) ). This equation is true for all $\eta \in \mathbb{R}^{d}$ if $\varphi \in \mathscr{S}\left(\mathbb{R}_{*}^{d}\right)$. By Lemma 4.3 there is $\widetilde{T} \in \mathscr{O}_{H}^{\prime}\left(\mathbb{R}^{d}\right)$ such that $T \varphi=\widetilde{T} \varphi$ for $\varphi \in \mathscr{S}\left(\mathbb{R}_{*}^{d}\right)$. This means that $L_{\widetilde{T}}(S)=S \star \widetilde{T}$ defines a Hadamard operator and $L(S) \varphi=L_{\widetilde{T}}(\varphi)$ for all $\varphi \in \mathscr{S}\left(\mathbb{R}_{*}^{d}\right)$. Therefore $L-L_{\widetilde{T}}$ is a Hadamard operator such that $\left(L-L_{\widetilde{T}}\right) S$ vanishes on $\mathscr{S}\left(\mathbb{R}_{*}^{d}\right)$ for all $S \in \mathscr{S}^{\prime}\left(\mathbb{R}^{d}\right)$, hence $\left(L-L_{\widetilde{T}}\right) x^{\alpha}=0$ for all $\alpha$ and therefore $L-L_{\widetilde{T}}=0$. Finally we have $T=L\left(\delta_{1}\right)=L_{\widetilde{T}}\left(\delta_{1}\right)=\widetilde{T}$. Therefore we have $L(S)=S \star T$ for all $S \in \mathscr{S}^{\prime}\left(\mathbb{R}^{d}\right)$.

\section{Final remarks}

In [11] the Hadamard operators in $\mathscr{D}^{\prime}\left(\mathbb{R}^{d}\right)$ were characterized. We can express the Main Theorem of [11] in the following way:

Theorem 5.1 The Hadamard operators on $\mathscr{D}^{\prime}\left(\mathbb{R}^{d}\right)$ are the operators of the form $S \mapsto S \star T$ where $T \in \mathscr{O}_{H}^{\prime}\left(\mathbb{R}_{*}^{d}\right)$ and $\operatorname{supp} T$ has positive distance to the coordinate hyperplanes.

This follows from the fact that for a distribution $T \in \mathscr{D}^{\prime}\left(\mathbb{R}^{d}\right)$, the support of which has positive distance of the coordinate hyperplanes, the conditions $T \in \mathscr{O}_{H}^{\prime}\left(\mathbb{R}^{d}\right)$ and $T \in \mathscr{O}_{H}^{\prime}\left(\mathbb{R}_{*}^{d}\right)$ coincide. For the definition of $\mathscr{O}_{H}^{\prime}\left(\mathbb{R}^{d}\right)$ see [11, Definition 3].

This implies:

Corollary 5.2 Every Hadamard operator on $\mathscr{D}^{\prime}\left(\mathbb{R}^{d}\right)$ maps $\mathscr{S}^{\prime}\left(\mathbb{R}^{d}\right)$ into $\mathscr{S}^{\prime}\left(\mathbb{R}^{d}\right)$.

By $\sigma(x)=\prod_{j} \frac{x_{j}}{\left|x_{j}\right|}$ we denote the signum of $x$. For $\alpha \in \mathbb{N}_{0}^{d}$ and $T=\theta^{\beta} \tau_{\beta}$ with $\left(|x|^{2 k}+|x|^{-2 k}\right) t_{\beta} \in L_{\infty}\left(\mathbb{R}^{d}\right)$ and $k$ large enough we define

$$
T\left(\frac{\sigma(x)}{x^{\alpha+1}}\right)=\int \tau_{\beta}(x)\left(\theta^{*}\right)^{\beta} \frac{\sigma(x)}{x^{\alpha+1}} d x .
$$

Therefore, using a proper representation, we can define $T\left(\frac{\sigma(x)}{x^{\alpha+1}}\right)$ for any $T \in$ $\mathscr{O}_{H}^{\prime}\left(\mathbb{R}_{*}^{d}\right)$. The definition does not depend on the representation, as the following result shows.

Theorem 5.3 If $T \in \mathscr{O}_{H}^{\prime}\left(\mathbb{R}_{*}^{d}\right)$ and $L(S):=S \star T$ the related Hadamard operator on $\mathscr{S}^{\prime}\left(\mathbb{R}^{d}\right)$, then the eigenvalues of $L$ with respect to $x^{\alpha}$ are $m_{\alpha}=T\left(\frac{\sigma(x)}{x^{\alpha+1}}\right)$. 
The Proof is the same as the proof of Theorem 4.2 in [11]. In a remark after the proof there it is pointed out that it holds in a very general context.

We could also, in analogy to Section 2, define

$\mathscr{O}_{H}\left(\mathbb{R}_{*}^{d}\right):=\left\{f \in C^{\infty}\left(\mathbb{R}_{*}^{d}\right): \exists k \in \mathbb{N}_{0} \forall \alpha \in \mathbb{N}_{0}^{d}: \sup _{x \in \mathbb{R}_{*}^{d}}\left|\theta^{(\alpha)} f(x)\right|\left(|x|^{2 k}+|x|^{-2 k}\right)<+\infty\right\}$.

Equipped with its natural locally convex topology $\mathscr{O}_{H}\left(\mathbb{R}_{*}^{d}\right)$ is the inductive limit of a sequence of Fréchet spaces, that is, an (LF)-space and we have

Lemma $5.4 \mathscr{O}_{H}^{\prime}\left(\mathbb{R}_{*}^{d}\right)$ is the dual space of $\mathscr{O}_{H}\left(\mathbb{R}_{*}^{d}\right)$.

This can be derived from Lemma 2.6 by use of the exponential transformation applied to all quadrants, or by direct verification. In this setting the term $T\left(\frac{\sigma(x)}{x^{\alpha+1}}\right)$ is properly defined.

\section{References}

[1] P. Domański, M. Langenbruch, Representation of multipliers on spaces of real analytic functions, Analysis 32 (2012), 137-162.

[2] P. Domański, M. Langenbruch, Algebra of multipliers on the space of real analytic functions of one variable, Studia Math. 212 (2012), 155-171.

[3] P. Domański, M. Langenbruch, Hadamard multipliers on spaces of real analytic functions, Adv. Math. 240 (2013), 575-612.

[4] P. Domański, M. Langenbruch, Surjectivity of Hadamard type operators on spaces of smooth functions, Rev. R. Acad. Cien. Serie A. Mat. (2018), doi.org/10.1007/s13398-018-0560-6.

[5] P. Domański, M. Langenbruch, D. Vogt, Hadamard type operators on spaces of real analytic functions in several variables, J. Funct. Anal. 269 (2015), 3868-3913.

[6] J. J. Duistermaat, J. A. C. Kolk, Distributions. Theory and Applications, Birkhäuser, Boston 2010.

[7] R. Meise, D. Vogt: Introduction to functional analysis, Clarendon Press, Oxford, (1997).

[8] L. Schwartz, Théorie des distributions I, Hermann, Paris 1957.

[9] L. Schwartz, Théorie des distributions II, Hermann, Paris 1959. 
[10] D. Vogt, Operators of Hadamard type on spaces of smooth functions, Math. Nachr., 288 (2015), 353-361.

[11] D. Vogt, Hadamard operators on $\mathscr{D}^{\prime}\left(\mathbb{R}^{d}\right)$, Studia Math. 237 (2017), 137-152.

[12] D. Vogt, Hadamard operators on $\mathscr{D}^{\prime}(\Omega)$, Math. Nachr. 290 (2017), 13741380 .

[13] D. Vogt, $\mathscr{E} \prime$ as an algebra by multiplicative convolution, Functiones et Approximatio (2018), DOI: 10.7169/facm/1719.

[14] D. Vogt, Surjectivity of Euler operators on temperate distributions, J. Math. Anal. Appl. 266 (2018), 1393-1399.

Bergische Universität Wuppertal, Dept. of Math., Gauß-Str. 20, D-42119 Wuppertal, Germany

e-mail:dvogt@math.uni-wuppertal.de 